\title{
Protein Transduction: A Novel Tool for Tissue Regeneration
}

\author{
M. Cristina Cardoso ${ }^{1, *}$ and \\ Heinrich Leonhardt ${ }^{1,2}$ \\ ${ }^{1}$ Max Delbrück Center for Molecular Medicine, \\ D-13125 Berlin, Germany \\ ${ }^{2}$ Department of Biology II, Ludwig Maximilians \\ University, D-80336 Munich, Germany
}

${ }^{*}$ Corresponding author

Tissue regeneration in humans is limited and excludes vitals organs like heart and brain. Transformation experiments with oncogenes like $T$ antigen have shown that retrodifferentiation of the respective cells is possible but hard to control. To bypass the risk of cancer formation a protein therapy approach has been developed. The transient delivery of proteins rather than genes could still induce terminally-differentiated cells to reenter the cell cycle. This approach takes advantage of protein-transducing domains that mediate the transfer of cargo proteins into cells. The goal of this brief review is to outline the basics of protein transduction and to discuss potential applications for tissue regeneration.

Key words: Cell cycle reentry/Protein therapy/ Retrodifferentiation/SV40 T antigen/TAT/VP22.

\section{Introduction: The Price of Differentiation}

Cellular differentiation, in general, is accompanied by withdrawal from the cell cycle (Figure 1). That generates on the one hand functional cells and tissues, which on the other hand are mostly not capable of regeneration. In terms of their respective proliferation potential, one can grossly distinguish three types of cells in mammals: the ones that are constantly dividing such as hematopoietic stem cells and epithelial cells; the ones that are mostly quiescent but can be stimulated to divide, including fibroblasts, hepatocytes, T and B cells; and the ones that do not divide, including neurons, heart and skeletal muscle cells. Particularly in the latter type, tissue repair after injury or disease is a major unsolved problem.

In recent years, progress at many fronts has indicated the existence of stem cells in adults that under some conditions can be persuaded to transdifferentiate into different cell lineages, unraveling a far greater plasticity than previously expected (Figure 1). However, these cells have a rather low proliferative potential and are therefore difficult to obtain in meaningful quantities. In parallel, studies on embryonic stem cells, which are by definition pluripotent, are raising hopes of finding the 'right way' to direct their differentiation into particular cell lineages. This approach is presently the subject of an intensive ethical debate.

\section{Ways to Regenerate a Tissue}

In principle, one could think of two ways to regenerate a tissue:

(i) induce 'neighboring' cells to proliferate and/or transdifferentiate;

(ii) transplant in vitro cultivated cells that can be differentiated into the particular lineage.

In nature, one can find examples of both strategies to replace body parts (see Pearson, 2001). Within some invertebrates such as the cnidarian Hydra, populations of 'totipotent' stem cells are present throughout their lives. Upon transection, stem cells near the place of injury are mobilized and respond to local cues, regrowing the missing body part. In some vertebrates, such as amphibian urodeles, differentiated cells near the amputated plane can start proliferating and become dedifferentiated. These so-called blastemal cells produce a growing cone of cells that progressively redifferentiate into the original or other cell types needed to reconstruct the missing body part (reviewed in Brockes, 1997). This cellular plasticity is now the focus of intensive research aimed at identifying the similarities and differences allowing regeneration in these organisms and failing in humans. Recent studies mixing mouse and urodele skeletal muscle revealed that the mouse cells could be stimulated to reenter the cell cycle (McGann et al., 2001; Velloso et al., 2001). Efforts are now concentrating on identifying the factors that mediate the activation of proliferation in mammalian skeletal muscle.

\section{Gene-Based Retrodifferentiation Approach}

Earlier studies have shown that the proliferation arrest of terminally differentiated mammalian cells is an actively maintained state that can, in principle, be reversed. Our knowledge of the molecular basis of cell cycle control 


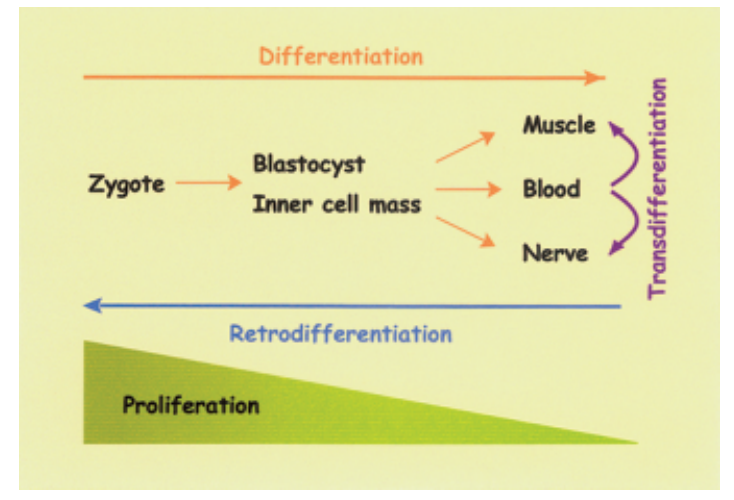

Fig. 1 Progressive Loss of Proliferative Capacity during Differentiation.

During the pre-implantation development cleavage cycles, from the totipotent zygote to the blastocyst stage, high levels of proliferation are maintained. Upon implantation and after birth, concomitant with the development of the different somatic lineages and increased differentiation, a slow but progressive loss of proliferation takes place. Several differentiated tissues such as heart muscle, skeletal muscle and neurons become post-mitotic, although in some cases limited populations of stem cells have been found. Recent studies have shown that some of these (stem) cells are able to differentiate into other cell lineages (transdifferentiation). In addition, expression of e.g. oncogenes, has been shown to be able to reverse the terminally differentiated state in some of these cells (retrodifferentiation).

during terminal differentiation is still limited, but several of the essential components and links have been identified using the skeletal muscle system (reviewed by Cardoso and Leonhardt, 1999). In mitogen-rich conditions, high levels of the cell cycle activator, cyclin D1, mediate repression of (i) retinoblastoma protein (pRb) growth suppressive activity and (ii) MyoD transactivation of the general cyclin-dependent kinase inhibitor p21 cip1 and muscle-specific genes, thus keeping myoblasts in a proliferative state. Mitogen depletion (or other inductive cues) induces the down-regulation of cyclin D1. This allows accumulation of under-phosphorylated $\mathrm{pRb}$, which represses the transcription of proliferation-associated genes by binding to E2F-DP transcription factor complex. In addition, release of cyclin D1 inhibition of MyoD transactivation leads to the induction of the muscle differentiation program and $\mathrm{p} 21^{\mathrm{cip} 1}$ accumulation accompanied by cell cycle withdrawal.

Regulated expression of oncogenes such as SV40 large $T$ antigen, which inhibits the $\mathrm{pRb}$ family of proteins, stimulates cell cycle reentry (Endo and Nadal-Ginard, 1989; Cardoso et al., 1993). Other viral oncogenes have also been shown, with different degrees of efficacy, to stimulate proliferation of differentiated cells.

Another approach has been the generation of transgenic mice harboring a temperature-sensitive SV40 large T antigen. Upon ex vivo cultivation at the permissive temperature, these cells proliferated and could be expanded in a manner dependent on active $T$ antigen and, at least in some cases, could be re-implanted de novo into mice and redifferentiated (reviewed in Obinata, 1997).

In a related approach, reversible immortalization and expansion of differentiated cells was achieved by viral delivery of an excisable SV40 large T antigen, which was later removed by bacteriophage Cre recombinase-mediated excision. Importantly, after excision, the cells reverted back to the differentiated phenotype (Li et al., 1997; Berghella et al., 1999; Kobayashi et al., 2000).

All these studies have in common that they are based on the delivery of the viral oncogene. Besides the hurdles of gene delivery itself, none of these genetic methods provides a good control over dose and time/duration of delivery. Most importantly, a single 'misguided' DNA molecule inserted in an actively transcribed region of the host chromosome could irreversibly transform the host and thus give rise to a deadly tumor raising serious safety concerns that at this moment seem to rule out any therapeutic applications.

\section{Protein Transduction: An Alternative to Genetic Manipulation}

Until now the applicability of gene delivery systems such as adenoviral vectors has been limited by problems to sustain a stable transgene expression over long enough time periods and by side effects like immunogenicity of viral vectors or potential malignant transformation of target cells (reviewed by Verma and Somia, 1997). Furthermore, the efficacy of infection and transfection methods is quite low in many differentiated cells.

The direct transfer of therapeutic proteins to target cells would avoid irreversible genetic modification (mutation) of targeted cells and would allow a better control over dose and treatment duration. Protein therapy would therefore be a very interesting alternative for a transient and non-transforming effect since proteins get naturally degraded and leave no traces behind. The fact that the plasma membrane is rather impermeable (cut-off around $600 \mathrm{Da}$ ) has made intracellular delivery of large molecules to cells without membrane disruption an impossible goal. In the last decade, the observation that some viral and cellular proteins could transverse the cellular membrane and be targeted to the cytoplasm and nucleus opened up totally new avenues to exploit protein transduction for intracellular therapies and functional studies in general.

The first case of a protein that could cross the lipid bilayer was the HIV-1 TAT protein (Frankel and Pabo, 1988; Green and Loewenstein, 1988). A little later, a Drosophila homeodomain transcription factor, Antennapedia, was shown to also translocate across cellular membranes (Joliot et al., 1991). Subsequent mapping identified short peptide sequences in these proteins, rich in basic amino acids that confer cellular internalization. The sequences have therefore been named protein transducting domains. 


\section{Protein Transducing Domains (PTD)}

PTD are peptide sequences from viral or cellular proteins that can transduce across the plasma membrane in a receptor-independent manner. The most well-studied and commonly used ones are: (i) HIV-1 TAT; (ii) HSV-1 VP22; (iii) Drosophila Antennapedia. In addition, other proteins have been reported to translocate across the membrane in this unconventional manner. The latter proteins will not be further mentioned here but several examples can be found in Prochiantz (2000) and Futaki et al. (2001).

In general, the PTD vector peptide is covalently bound to the cargo. Internalization has been in all cases shown to occur at $37^{\circ} \mathrm{C}$ and at $4{ }^{\circ} \mathrm{C}$, and to be receptor independent. To date, all cell types have been shown to be transducible in vitro and in vivo. Upon import the complex, in principle, does not dissociate, neither does it enter the endocytic pathway. Transduction occurs in a concentration-dependent manner and within a few minutes, allowing intracellular delivery of biomolecules in a timeand dose-controlled manner.

The precise mechanism of PTD-mediated translocation through the membrane is not known but several studies have investigated the minimal transduction domains and potential structural requirements. To date, no clear structural requirements have been identified.

Extensive mutagenesis studies have identified the essential residues required for translocation in the 16 amino acid-long Antennapedia peptide. In addition, a multitude of cargoes have been cross-linked and delivered intracellularly by this PTD. A shortcoming of this PTD is its inability to internalize cargoes of sizes around or above $30 \mathrm{kDa}$, as well as its relatively lower efficiency when compared with the TAT PTD. Work on Antennapedia PTD has been the subject of several excellent reviews (Prochiantz, 1996, 2000) and will not be further reviewed here.

\section{HIV-1 TAT}

TAT is a 101 amino acid immediate-early HIV protein that is a powerful transactivator of gene expression and essential for viral replication (reviewed in Jeang et al., 1999). As mentioned above, it was the first protein reported to be able to translocate through the plasma membrane and enter the nucleus (Frankel and Pabo, 1988; Green and Loewenstein, 1988). Its translocation ability was shown to be mediated by an 11 amino acid-basic peptide [YGRKKRRQRRR] (Vives et al., 1997). Subsequently, an ever-increasing number of fusion proteins and other cargoes ranging from small molecules, peptides, anti-sense oligonucleotides to 200 nm-large liposomes, have been successfully transduced via this PTD and other arginine- or lysine-rich peptides (reviewed in Wadia and Dowdy, 2002). Until now no size limit has been established for this PTD.

The small size of this peptide and its high content of arginines and lysines have prompted in the last years a search for naturally or artificially improved PTDs. A collection of the best PTDs from studies where the efficiency of different PTDs was compared is summarized in
Table 1. As can be seen in the Table, a direct comparison between reports is difficult since the individual studies were done using different cells (a few also on animals) and in some cases labeled peptides were tested on 'inline' fusion proteins or proteins conjugated to the peptides in different ways. Nevertheless, in many cases homopolymers of arginine or lysine longer than 7 residues transduce more efficiently than the TAT peptide itself.

The proposal that the guanidinium group of the arginine residues is required for transduction (Wender et al., 2000) does not seem to be an absolute requirement since polylysine PTDs have been shown to transduce even more efficiently than TAT or polyarginine PTDs (Mai et al., 2002).

Many TAT fusion proteins have been shown to date to enter the cell and retain functionality. In fact, it has even been proposed that unfolded denatured proteins are better transported across the lipid bilayer than native ones and can regain their active conformation inside the cell (Nagahara et al., 1998). Although the unfolded state might help internalization, it is also not an absolute requirement since other studies have successfully internalized native proteins (Mi et al., 2000). Thus, protein transduction technology has shown quite a degree of flexibility making it somehow difficult to select a priori which strategy or PTD is going to work best in a particular case.

One potential pitfall of these PTDs that has not received enough attention is the tendency of these peptides to accumulate in the nucleolus (Vives et al., 1997; Futaki et al., 2001). This in principle unwanted feature is, at least in some cases, dominant over other localization sequences and can prevent the correct functioning of the fused peptides or proteins (our unpublished results). A possible way to circumvent this problem is to engineer in vivo 'cleavable' peptides, which upon import into the intercellular milieu will then separate from the vector peptide. This approach has been successfully reported with the Antennapedia peptide. The authors have linked the Antennapedia peptide via a disulfide bond to the cargo. This bridge is not stable in the cytoplasm due to the presence of high levels of glutathione and becomes reduced in about an hour, liberating the cargo from the vector peptide (Theodore et al., 1995; Prochiantz, 1996). This removal of the PTD also prevents later extrusion of the cargo out of the cell. Definitely, further work needs to be done in order to target macromolecules to the desired subcellular compartments of their action and to prevent mistargeting of the cargo caused by the attached PTDs.

Recently, a novel protein delivery system was described that is based on the HSV-1 protein VP22, which is exported from cells by a Golgi-independent mechanism and then spreads to neighboring cells (Elliott and O'Hare, 1997).

\section{VP22}

The $38 \mathrm{kDa}$ viral phosphoprotein VP22 is a structural component of HSV-1 located between capsid and tegu- 
Table 1 'Top' Natural and Artificial PTDs.

\begin{tabular}{llll}
\hline PTD & Uptake as & Tested in & Reference \\
\hline RRQRRTSKLMKR & peptide, fusion protein and conjugate & cell culture and animals & Mi et al. (2000) \\
RRRRRRRRR & labeled peptide & cell culture & Wender et al. (2000) \\
YARAAARQARA & labeled peptide & cell culture and animals & Ho et al. (2001) \\
RRRRRRRR & peptide and cross-linked protein & cell culture & Futaki et al. (2001) \\
RRRRRRRRR & fusion protein & cell culture & Han et al. (2001) \\
KKKKKKKK & protein conjugate & cell culture & Mai et al. (2002) \\
KKKKKKKKK & fusion protein & cell culture and animals & Park et al. (2002) \\
\hline
\end{tabular}

Collection of the most efficient PTDs from comparative studies where the uptake of different peptides was tested.

aBiotinylated peptide conjugated to avidin-linked $\beta$-galactosidase or fluorochrome.

${ }^{\mathrm{b}} \mathrm{D}$-isomers were more efficient than $\mathrm{L}$-isomers.

'Biotinylated peptide conjugated to streptavidin-linked $\beta$-galactosidase.

dUptake efficiency highly dependent on the cell line and enhanced by dextran sulfate.

Table 2 Summary of Proteins Transduced via Fusion to VP22.

\begin{tabular}{|c|c|c|c|}
\hline Protein fused to VP22 & Physiological effect & Tested in & Reference \\
\hline p53 - n - n & induction of apoptosis & cell culture & Phelan et al. (1998) \\
\hline HSV thymidine kinase & suicide cell death & cell culture and animals $\mathrm{s}^{\mathrm{a}, \mathrm{b}}$ & Dilber et al. (1999) \\
\hline HSV thymidine kinase & suicide cell death & cell culturea & Liu et al. (2001) \\
\hline p53 - n - n - n & induction of apoptosis & cell culture and animals ${ }^{b}$ & Wills et al. (2001) \\
\hline cytosine deaminase & suicide cell death & cell culture ${ }^{\mathrm{b}}$ & Wybranietz et al. (2001) \\
\hline SV40 T antigen & cell cycle reinduction & cell culture ${ }^{a}$ & Derer et al. (2002) \\
\hline p53 & induction of apoptosis & cell culture and animals ${ }^{\mathrm{a}, \mathrm{b}}$ & Zender et al. (2002) \\
\hline
\end{tabular}

Proteins such as $\beta$-galactosidase or GFP are not included since they are only used as reporters to test efficiency.

aCoculture of VP22-producing cells with recipient cells.

bVP22 fusion encoded in viral vectors.

ment. Its function in the viral life cycle is not clear. The Cterminal half of the protein shares extensive homology with tegument VP22 proteins from other herpes viruses (Normand et al., 2001; Ren et al., 2001). This protein domain can be overproduced in bacteria and has been shown in the presence of oligonucleotides to produce spherical particles (named vectosomes) that can transport these oligonucleotides into mammalian cells. Furthermore, light activation can induce the release of the cargo within seconds (Normand et al., 2001).

VP22 has a complex pattern of localization in expressing cells. It is first detected in the cytoplasm where it can bind and reorganize microtubules. When cells enter mitosis, it binds to chromatin and after cell division remains in the nucleus of the daughter cells (Elliott and O'Hare, 2000). Very little is known about intercellular transport or the export and import mechanisms involved in the VP22 intercellular spreading process. Recent studies have mapped the protein domains required for the different subcellular localizations as well as for intercellular spreading (Aints et al., 2001; Ren et al., 2001; Martin et al., 2002).

As summarized in Table 2, different fusion proteins have been generated and shown to spread and retain their physiological effects. Most reports have concentrated on anti-tumor therapies either by induction of apoptosis upon delivery of functional p53 fused to VP22 (Phelan et al., 1998; Wills et al., 2001; Zender et al., 2002) or by delivering enzymes (thymidine kinase or cytosine deaminase) that allow the conversion of commonly used prodrugs into toxic compounds thereby inducing cell death (Dilber et al., 1999; Liu et al., 2001; Wybranietz et al., 2001). In some cases, coculture of VP22-producing transfected cells and recipient cells was used. Other studies tested whether the efficiency of viral delivery would be enhanced by the subsequent ability of the produced proteins to spread into non-infected cells via the VP22 moiety.

Due to the intended application in tumor therapy, most studies to date have investigated the ability of VP22 to spread between proliferating cells. We have further established the ability of VP22 fusion proteins to spread into terminally differentiated cells (Derer et al., 1999, 2002), which is the prerequisite for any potential application in tissue regeneration.

\section{Direct Protein Delivery as a Tool for Tissue Regeneration}

A desirable regenerating agent should be able to induce otherwise terminally differentiated cells to proliferate for some time and disappear afterward leaving no traces behind and permitting subsequent cellular redifferentiation. 


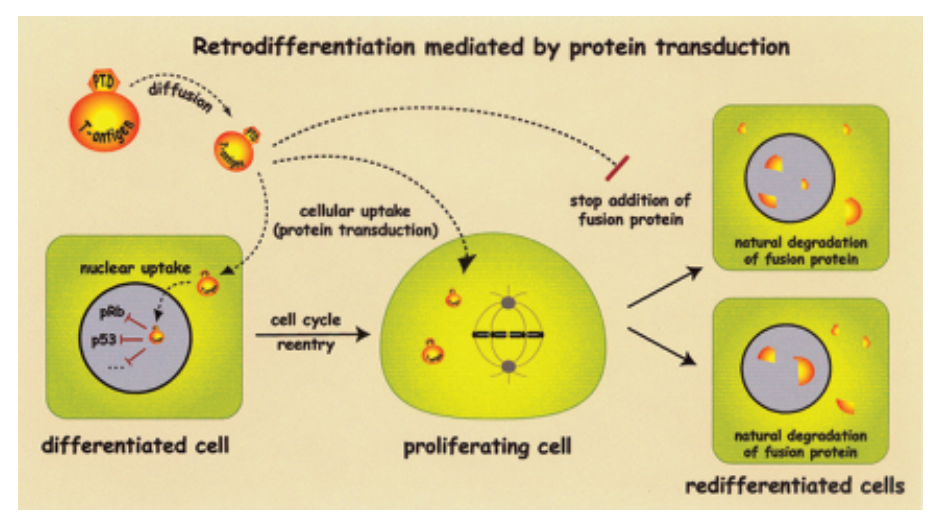

Fig. 2 Protein Transduction as a Tool to Transiently Induce Retrodifferentiation.

The outlined strategy is based on some recent results obtained with an SV40 T antigen fusion (Derer et al., 2002). Chimeric proteins composed of PTD and SV40 T antigen (PTD-T antigen) transverse the cell membrane and enter the nucleus of terminally differentiated cells. The multifunctional viral T antigen protein releases the cell cycle arrest actively maintained by the retinoblastoma family of proteins and prevents apoptosis mediated by p53, thereby allowing expression of cell cycle activators and induction of cellular proliferation. Once the supply of fresh PTD-T antigen is discontinued, the remaining fusion protein gets naturally degraded and leaves no traces behind, allowing subsequent redifferentiation of the cells.

Since in most cases of unscheduled proliferation cells activate their apoptosis program, the ideal agent should also be able to prevent the programmed cell death. It would in addition be desirable to have an agent that acts topically so as to allow regeneration of the specific tissue and not at other body parts. For that purpose, either a very rapid uptake of the regenerating agent, or ex vivo application, followed by grafting of the expanded and differentiated cells, would be two possible alternatives. Most factors that can induce differentiated cells to reenter the cell cycle are powerful oncogenes, i.e., they can induce tumors. This capacity of oncogenes to permanently transform cells that could then give rise to tumors constitutes a serious risk and argues against gene-based approaches. Therefore, a transient and non-transforming approach such as protein transduction, i.e., direct delivery of the oncogene product rather than the gene itself, seems to be the method of choice.

The proof-of-concept that protein transduction can be used to induce proliferation in terminally differentiated cells was recently obtained (Derer et al., 2002). In this study, cells overproducing a fusion of VP22 with SV40 large $T$ antigen were cocultured with differentiated skeletal muscle cells. VP22 was shown to mediate the trafficking of SV40 large T antigen into the differentiated myotubes where it accumulated in the nucleus. The fusion protein was also able to activate cell cycle reentry in these differentiated cells.

SV 40 large $T$ antigen combines the ability to release the cell cycle block maintained by the retinoblastoma family of tumor suppressors, to block apoptosis (reviewed in Ali and DeCaprio, 2001) and possibly even to activate telomerase, preventing telomere shortening (Foddis et al., 2002). Furthermore, gene-based approaches have indicated that upon removal of $\mathrm{T}$ antigen, cells revert back to the differentiated phenotype (Li et al.,
1997; Obinata, 1997; Berghella et al., 1999; Kobayashi et al. , 2000). Therefore, this oncoprotein is an ideal candidate for a regenerating agent.

Another advantage of the direct delivery approach is the fact that it allows for easy combinatorial therapies using a mixture of different factors, which would be rather difficult to achieve with gene-based methods. A potential pitfall of longer term applications is the likely development of an immune reaction to the administered agent. Methods of circumventing this problem are already in use, and involve, in addition to immune suppression, generation of functional mutants of the delivered protein or homologs from other species (reviewed in Ford et al., 2001).

Certainly, several steps need to be optimized before the therapeutical application of this protein transduction approach for tissue regeneration. Based on our recent results using a T antigen fusion protein (Derer et al., 2002) we propose the approach for tissue regeneration outlined in Figure 2 that could in principle be used directly on damaged tissues or indirectly by expanding cells ex vivo for later engrafting. Purified PTD-T antigen is added to the cell culture medium in ex vivo cultures or applied to the damaged tissue, where it crosses the plasma membrane and enters the nucleus. There SV40 large T antigen binds $\mathrm{pRb}$ and $\mathrm{p53}$, among other cellular proteins, and induces cellular proliferation. Upon attaining sufficient cell numbers, addition of PTD-T antigen is stopped. Over time the remaining fusion protein gets naturally degraded allowing the cells to redifferentiate and to functionally replace the damaged tissue.

In the near future, the purification of PTD-T antigen fusion proteins, as well as other potential candidates and mixtures thereof, is necessary to evaluate and optimize their potential to elicit transient expansion of differentiated cells in vitro and in vivo. In parallel, the feasibility of us- 
ing PTDs that get cleaved off within the cell should be tested for this approach. Furthermore, fast and efficient transduction might allow topical administration of the chosen regeneration agent. Finally, estimating and engineering the half-life of the regeneration factor in different cellular and extracellular environments will be necessary. Although a lot of work still lies ahead, the potential of this strategy amply justifies the effort.

\section{References}

Aints, A., Guven, H., Gahrton, G., Smith, C. I., and Dilber, M. S. (2001). Mapping of herpes simplex virus-1 VP22 functional domains for inter- and subcellular protein targeting. Gene Ther. 8, 1051-1056.

Ali, S. H., and DeCaprio, J. A. (2001). Cellular transformation by SV40 large T antigen: interaction with host proteins. Semin. Cancer Biol. 11, 15-23.

Berghella, L., De Angelis, L., Coletta, M., Berarducci, B., Sonnino, C., Salvatori, G., Anthonissen, C., Cooper, R., ButlerBrowne, G. S., Mouly, V. et al. (1999). Reversible immortalization of human myogenic cells by site-specific excision of a retrovirally transferred oncogene. Hum. Gene Ther. 10, 1607 1617.

Brockes, J. P. (1997). Amphibian limb regeneration: rebuilding a complex structure. Science 276, 81-87.

Cardoso, M. C., and Leonhardt, H. (1999). Differentiation, development and programmed cell death. In: The Molecular Basis of Cell Cycle and Growth Control, G. Stein, R. Baserga, D. Denhardt, and A. Giordano, eds. (New York, USA: John Wiley \& Sons), pp. 305-347.

Cardoso, M. C., Leonhardt, H., and Nadal-Ginard, B. (1993). Reversal of terminal differentiation and control of DNA replication: cyclin A and Cdk2 specifically localize at subnuclear sites of DNA replication. Cell 74, 979-992.

Derer, W., Easwaran, H. P., Knopf, C. W., Leonhardt, H., and Cardoso, M. C. (1999). Direct protein transfer to terminally differentiated muscle cells. J. Mol. Med. 77, 609-613.

Derer, W., Easwaran, H. P., Leonhardt, H., and Cardoso, M. C. (2002). A novel approach to induce cell cycle reentry in terminally differentiated muscle cells. FASEB J. 16, 132-133.

Dilber, M. S., Phelan, A., Aints, A., Mohamed, A. J., Elliott, G., Smith, C. I. E., and O'Hare, P. (1999). Intercellular delivery of thymidine kinase prodrug activating enzyme by the herpes simplex virus protein, VP22. Gene Ther. 6, 12-21.

Elliott, G., and O'Hare, P. (1997). Intercellular trafficking and protein delivery by a herpes virus structural protein. Cell 88 , 223-233.

Elliott, G., and O'Hare, P. (2000). Cytoplasm-to-nucleus translocation of a herpesvirus tegument protein during cell division. J. Virol. 74, 2131-2141.

Endo, T., and Nadal-Ginard, B. (1989). SV40 large T-antigen induces reentry of terminally differentiated myotubes into the cell cycle. In: The Cellular and Molecular Biology of Muscle Development, L. H. Kedes, and F. E. Stockdale, eds. (New York, USA: Alan R. Liss, Inc.), pp. 95-104.

Foddis, R., De Rienzo, A., Broccoli, D., Bocchetta, M., Stekala, E., Rizzo, P., Tosolini, A., Grobelny, J. V., Jhanwar, S. C., Pass, H. I. et al. (2002). SV40 infection induces telomerase activity in human mesothelial cells. Oncogene 21, 1434-1442.

Ford, K. G., Souberbielle, B. E., Darling, D., and Farzaneh, F. (2001). Protein transduction: an alternative to genetic intervention? Gene Ther. 8, 1-4.

Frankel, A. D., and Pabo, C. O. (1988). Cellular uptake of the tat protein from human immunodeficiency virus. Cell 55, 1189-1193.

Futaki, S., Suzuki, T., Ohashi, W., Yagami, T., Tanaka, S., Ueda, K., and Sugiura, Y. (2001). Arginine-rich peptides. An abundant source of membrane-permeable peptides having potential as carriers for intracellular protein delivery. J. Biol. Chem. 276, 5836-5840.

Green, M., and Loewenstein, P. M. (1988). Autonomous functional domains of chemically synthesized human immunodeficiency virus tat trans-activator protein. Cell 55, 1179-1188.

Han, K., Jeon, M. J., Kim, S. H., Ki, D., Bahn, J. H., Lee, K. S., Park, J., and Choi, S. Y. (2001). Efficient intracellular delivery of an exogenous protein GFP with genetically fused basic oligopeptides. Mol. Cells 12, 267-271.

Ho, A., Schwarze, S. R., Mermelstein, S. J., Waksman, G., and Dowdy, S. F. (2001). Synthetic protein transduction domains: enhanced transduction potential in vitro and in vivo. Cancer Res. 61, 474-477.

Jeang, K. T., Xiao, H., and Rich, E. A. (1999). Multifaceted activities of the HIV-1 transactivator of transcription, Tat. J. Biol. Chem. 274, 28837-28840.

Joliot, A., Pernelle, C., Deagostini-Bazin, H., and Prochiantz, A. (1991). Antennapedia homeobox peptide regulates neural morphogenesis. Proc. Natl. Acad. Sci. USA 88, 1864-1868.

Kobayashi, N., Fujiwara, T., Westerman, K. A., Inoue, Y., Sakaguchi, M., Noguchi, H., Miyazaki, M., Cai, J., Tanaka, N., Fox, I. J., and Leboulch, P. (2000). Prevention of acute liver failure in rats with reversibly immortalized human hepatocytes. Science 287, 1258-1262.

Li, L. P., Schlag, P. M., and Blankenstein, T. (1997). Transient expression of SV 40 large T antigen by Cre/LoxP-mediated sitespecific deletion in primary human tumor cells. Hum. Gene Ther. 8, 1695-1700.

Liu, C. S., Kong, B., Xia, H. H., Ellem, K. A., and Wei, M. Q. (2001). VP22 enhanced intercellular trafficking of HSV thymidine kinase reduced the level of ganciclovir needed to cause suicide cell death. J. Gene Med. 3, 145-152.

Mai, J. C., Shen, H., Watkins, S. C., Cheng, T., and Robbins, P. D. (2002). Efficiency of protein transduction is cell type-dependent and is enhanced by dextran sulfate. J. Biol. Chem. 28, 30208-30218.

Martin, A., O'Hare, P., McLauchlan, J., and Elliott, G. (2002). Herpes simplex virus tegument protein VP22 contains overlapping domains for cytoplasmic localization, microtubule interaction, and chromatin binding. J. Virol. 76, 4961-4970.

McGann, C. J., Odelberg, S. J., and Keating, M. T. (2001). Mammalian myotube dedifferentiation induced by newt regeneration extract. Proc. Natl. Acad. Sci. USA 98, 13699-13704.

Mi, Z., Mai, J., Lu, X., and Robbins, P. D. (2000). Characterization of a class of cationic peptides able to facilitate efficient protein transduction in vitro and in vivo. Mol. Ther. 2, 339-347.

Nagahara, H., Vocero-Akbani, A. M., Snyder, E. L., Ho, A., Latham, D. G., Lissy, N. A., Becker-Hapak, M., Ezhevsky, S. A., and Dowdy, S. F. (1998). Transduction of full-length TAT fusion proteins into mammalian cells: TAT-p27Kip1 induces cell migration. Nature Med. 4, 1449-1452.

Normand, N., van Leeuwen, H., and O'Hare, P. (2001). Particle formation by a conserved domain of the herpes simplex virus protein VP22 facilitating protein and nucleic acid delivery. J. Biol. Chem. 276, $15042-15050$.

Obinata, M. (1997). Conditionally immortalized cell lines with differentiated functions established from temperature-sensitive T-antigen transgenic mice. Genes Cells 2, 235-244.

Park, J., Ryu, J., Jin, L. H., Bahn, J. H., Kim, J. A., Yoon, C. S., Kim, D. W., Han, K. H., Eum, W. S., Kwon, H. Y. et al. (2002). 9polylysine protein transduction domain: enhanced penetra- 
tion efficiency of superoxide dismutase into mammalian cells and skin. Mol. Cells 13, 202-208.

Pearson, H. (2001). The regeneration gap. Nature 414, 388-390. Phelan, A., Elliott, G., and O'Hare, P. (1998). Intercellular delivery of functional p53 by the herpesvirus protein VP22. Nature Biotechnol. 16, 440-443.

Prochiantz, A. (1996). Getting hydrophilic compounds into cells: lessons from homeopeptides. Curr. Opin. Neurobiol. 6, $629-634$

Prochiantz, A. (2000). Messenger proteins: homeoproteins, TAT and others. Curr. Opin. Cell Biol. 12, 400-406.

Ren, X., Harms, J. S., and Splitter, G. A. (2001). Bovine herpesvirus 1 tegument protein VP22 interacts with histones, and the carboxyl terminus of VP22 is required for nuclear localization. J. Virol. 75, $8251-8258$.

Theodore, L., Derossi, D., Chassaing, G., Llirbat, B., Kubes, M., Jordan, P., Chneiweiss, H., Godement, P., and Prochiantz, A. (1995). Intraneuronal delivery of protein kinase $C$ pseudosubstrate leads to growth cone collapse. J. Neurosci. 15, $7158-$ 7167.

Velloso, C. P., Simon, A., and Brockes, J. P. (2001). Mammalian postmitotic nuclei reenter the cell cycle after serum stimulation in newt/mouse hybrid myotubes. Curr. Biol. 11, 855-858.

Verma, I. M., and Somia, N. (1997). Gene therapy - promises, problems and prospects. Nature 389, 239-242.
Vives, E., Brodin, P., and Lebleu, B. (1997). A truncated HIV-1 Tat protein basic domain rapidly translocates through the plasma membrane and accumulates in the cell nucleus. J. Biol. Chem. $272,16010-16017$.

Wadia, J. S., and Dowdy, S. F. (2002). Protein transduction technology. Curr. Opin. Biotechnol. 13, 52-56.

Wender, P. A., Mitchell, D. J., Pattabiraman, K., Pelkey, E. T., Steinman, L., and Rothbard, J. B. (2000). The design, synthesis, and evaluation of molecules that enable or enhance cellular uptake: peptoid molecular transporters. Proc. Natl. Acad. Sci. USA 97, 13003-13008.

Wills, K. N., Atencio, I. A., Avanzini, J. B., Neuteboom, S., Phelan, A., Philopena, J., Sutjipto, S., Vaillancourt, M. T., Wen, S. F., Ralston, R. O., and Johnson, D. E. (2001). Intratumoral spread and increased efficacy of a p53-VP22 fusion protein expressed by a recombinant adenovirus. J. Virol. 75, 8733-8741.

Wybranietz, W. A., Gross, C. D., Phelan, A., O'Hare, P., Spiegel, M., Graeper, F., Bitzer, M., Stahler, P., Gregor, M., and Lauer, U. M. (2001). Enhanced suicide gene effect by adenoviral transduction of a VP22-cytosine deaminase (CD) fusion gene. Gene Ther. 8, 1654-1664.

Zender, L., Kuhnel, F., Kock, R., Manns, M., and Kubicka, S. (2002). VP22-mediated intercellular transport of p53 in hepatoma cells in vitro and in vivo. Cancer Gene Ther. 9, $489-496$. 